\title{
INSTRUCTIONS FOR AUTHORS
}

\section{Submission of papers}

Authors are encouraged to submit their original manuscripts online via the website: http://www.JMech.org.tw, where electronic submission and complete instructions for the preparation of manuscripts can be found.

The Journal employs a peer review system in the processing of manuscripts submitted for publication. Each manuscript is sent to reviewers (usually two or more) who are experts in the related fields. Decision as to the publication of the paper is based on the opinions expressed by the reviewers and the judgment of the Editorial board. Reviewers' suggestions for the revision of the manuscript are passed on to the author(s), who is entitled to make use of them or rebut them as he or she sees fit.

If there are any questions with regard to manuscript submission, please contact: kathy@iam.ntu.edu.tw

\section{Manuscript preparation}

Papers should conform to the following instructions:

- Language: The manuscript should be written in good English. It should have been carefully checked for clarity, conciseness, correctness of grammar, and typographical errors. Manuscripts should be typed and double-spaced with ample margin on one side of $21 \times 30 \mathrm{~cm}$ sheets (A4 format).

- Length: A full length paper or review including figures and tables should not normally exceed 4 pages. For a rough estimate, count 3 manuscript pages per printed page and 4 onecolumn figures per printed page. Space for figures, tables, and references lists, all of which are highly variable, should be estimated by comparison to closely similar material published in the Journal.

- Format: The main divisions are suggested to be arranged as follows: 1. Title page (containing: article, title, author (s), affiliation (s), and corresponding author's address, phone number, fax number and email address); 2. Abstract (of 200 words or less); 3. Keywords (of 4 or less); 4. Main text (containing: introduction, methods of solution, results and discussion, conclusion): 5. Acknowledgements; 6. Appendices; 7. References; 8. Tables; 9. Figure captions; 10. Figures. Abstracts are not required for short papers.

- Figures: All photographs, charts and diagrams are to be referred to as "Figures". Captions to figures should be typed consecutively on a separate page (s) at the end of the paper. The preferred format for figure files is .eps or tiff at a resolution of 1200 dpi for lines, 600 dpi for greyscale and 300 dpi for colour (which preferably should also be in CMYK - cyan magenta yellow black - format). Colour art is free of charge for online publication. If figures will be printed in black and white, please ensure that the main information will be visible and do not refer to colour in the text.

- Tables: Tables should be typed as part of the text, but in such a way as to avoid confusion with the text. Authors should try to ensure that a single table does not overlay on to the next page. All tables should have headings and be numbered.
- Units: Use of the international system units (SI units) is obligatory. Wherever possible, equations should be written in dimension form.

- Equations: Mathematical expressions should be consecutively numbered throughout the body of the paper at the right-hand margin in parentheses. Numbering starts anew with each appendix: Appendix A: (A1), (A2), etc., Appendix B: (B1), (B2), etc. Equation numbers mentioned in the text should be enclosed in parentheses, i.e. Eq. (1), Eqs. (1), (2).

- References: References should be indicated in square brackets according to the order of appearances in the text, i.e. [1], [2-4]. The full list should be collected at the end of the paper in numerical order. Examples of layout of references are given below.

1. Brown, H. E., Amstead, B. H. and Short, E., "Temperature and Velocity Distribution and Transfer of Heat in a Liquid Metal," J. Heat Transfer, 79, pp. 279-285 (1957).

2. Zienkiewicz, O.C., The Finite Element Method, 3rd Edition, McGraw-Hill, Maiden Head, England, pp. 45-48 (1977).

3. Chen, W. H. and Wu, C. W., "On Elastodynamic Fracture Mechanics Analysis of Bi-Material Structures Using Finite Element Method," Proc. 4th Conf. on Theo. Appl. Mech., Taiwan, R.O.C., pp. 147-166 (1980).

4. Kobayashi, H., "Optimization of Elastic Structure," M.S. Thesis, Dept. of Aeronautics and Astronautics, Mass. Inst. Tech., Mass., U.S.A. (1972).

\section{After acceptance}

The corresponding author will be notified by the Editor-inChief of the Journal upon acceptance of the article and invited to supply an electronic version of the accepted manuscript. In the course of the production process, the corresponding author will be asked to transfer the copyright of the article to the Society. This transfer will ensure the widest possible dissemination of information.

\section{Page charges}

There will be no page charges for contributions from outside of Taiwan.

For contributions from Taiwan, a regular page charge of NT $\$ 500$ per page will be assessed for articles within 8 published pages (full length papers) or 4 published pages (short papers). In addition, an excess page fee of NT $\$ 2,000$ per each exceeding page will also be charged to the author (s).

\section{PDF offprint}

An author is entitled to a PDF offprint of the published paper free of charge. The PDF offprint will be sent to the corresponding author at the email address supplied on submission. Print offprints may be ordered separately (in multiples of 50). 
599. Reflection of Plane Waves in a Rotating Temperature-Dependent Thermoelastic Solid with Diffusion

\section{B. Singh, L. Singh, S. Deswal}

607. Flow Field in a Skimming Flow Over a Vertical Drop Without End-Sill

Chang Lin, S.-C. Hsieh, W.-J. Lin, S.-H. Chou, R. V. Raikar

627. Modified Characteristics-Based Schemes for Compressible Flow Past an Airfoil

\section{A. A. Orang, A. Paykani}

637. Quasi-Steady Flow Dynamics Study of Human Aortic Valve with Numerical Techniques

\section{H.-H. Vu, C.-H. Hsu}

647. Modeling and Simulation of Bladder Power Pump Driven by External Electromagnet

\section{Li, T. Guan, W.-J. Huang, C.-H. Liang}

657. Compression Behavior of Annular Elastic Layers Bonded Between Rigid Plates

\section{H.C. Tsai}

665. An Amplification Factor to Enhance Stability for Structure-Dependent Integration Method

\section{S.-Y. Chang}

677. Size and Shape Effects on Strength and Ultimate Strain in FRP Confined Rectangular Concrete Columns

\section{F.-Y. Yeh, K.-C. Chang}

691. Optimal Design of a Vibration-Based Electromagnetic Energy Harvester Using a Simulated Annealing Algorithm

M.-C. Chiu, Y.-C. Chang, L.-J. Yeh, C.-H. Chung

701. Numerical Study of the Influence of Combustion Models and Kinetic Schemes When Predicting the Diffusion Flames

\section{A. Khelil, S. Nechad, H. Naji, L. Loukarfi, M. Braikia, M. Beriache}

715. The Numerical Method as Applied to Impact Resistance Analysis of Ogival Nose Projectiles on $6061-T 651$ Aluminum Plates Y.-L. Chen, H.-C. Chen

\section{Technical Note}

N13. Investigation of the Convection Term Discretization Schemes for a Force-Generated Ring-Vortex

\section{N. M. Nouri, S. Yekani Motlagh, M. Navidbakhsh, E. Yasari}

